\title{
Recent Advances in Systemic Treatments for HER-2 Positive Advanced Gastric Cancer
}

\author{
Seda Kahraman (D) \\ Suayib Yalcin (iD) ${ }^{2}$ \\ 'Yıldırım Beyazıt University Faculty of \\ Medicine, Department of Medical \\ Oncology, Ankara, Turkey; ${ }^{2}$ Hacettepe \\ University Institute of Cancer, \\ Department of Medical Oncology, \\ Ankara, Turkey
}

\begin{abstract}
Gastric cancer (GC) is the fifth most common cancer worldwide. Despite recent improvements in treatment quality and options, advanced gastric cancer remains one of the hardest to cure cancers, with a median overall survival (OS) of 10-12 months and a 5-year OS of approximately $5-20 \%$. There is an unmet need for further efforts to palliate diseaserelated symptoms, improve quality of life, increase tumor response rate, and prolong progression free and overall survival while balancing the toxicities of therapy. The most common type of GC is adenocarcinoma, which demonstrates morphological, biological, and clinical heterogeneity. A plethora of genomic alterations and the activation of numerous molecular pathways including human epidermal growth receptor 2 (HER2), epidermal growth factor receptor (EGFR), fibroblast growth factor receptor-2 (FGFR2), mesenchymal epidermal transforming factor receptor (MET), and the phosphatidylinositol 3-kinase (PI3K)/ mammalian target of rapamycin (mTOR) are responsible for the complex heterogeneity of GC. Efforts to validate the therapeutic effects of inhibiting some of these aberrantly expressed pathways have failed to lead to a clinically meaningful outcome apart from the overexpression/amplification of the HER2 gene, inhibition of which has had a significant impact on clinical practice. The only available biomarkers to guide the effective treatment of patients with advanced GC are HER2 overexpression, MSI/PD-L1 status, and FGFR alterations. Various anti-HER2 agents have been evaluated after the success of the ToGA trial, but none led to a significant enough clinical improvement to be considered a viable alternative for HER2-targeted therapy in advanced GC until the global Keynote-811 trial, which added pembrolizumab to trastuzumab in combination with chemotherapy. This combination demonstrated a survival advantage for the first time in the 11 years since ToGA. Trastuzumab deruxtecan (T-DXd) was also found to be effective in patients who had already received $>2$ previous lines of treatment. Despite these promising avenues, the optimal management of HER-2 positive GC still requires further development.
\end{abstract}

Keywords: gastric cancer, HER-2, trastuzumab, targeted therapy

\section{Introduction}

Gastric cancer (GC) is the fifth most common cancer worldwide. ${ }^{1}$ GC has a very heterogeneous morphologic, biologic and clinical nature. The most common type of GC is adenocarcinoma. Of the several morphology-based classification systems that have been proposed, the World Health Organization (WHO) (papillary, tubular, mucinous, and poorly cohesive (PCC-NOS)/signet ring) ${ }^{2}$ the Lauren (intestinal, diffuse, and mixed) classifications are the most commonly preferred. ${ }^{3}$ However, conventional morphologybased classification systems are unable to reflect the molecular heterogeneity of GC and are therefore not a sufficient guide for molecularly targeted treatments based on precision
Correspondence: Suayib Yalcin

Hacettepe University Institute of Cancer, Department of Medical Oncology, Sihhiye, Ankara, Turkey

Tel +90-5053780639

Email syalcin@hacettepe.edu.tr 
oncology results. A special molecular/genetic classification system can better define the genetic landscape of $\mathrm{GC}$, and may be helpful to further understand, prevent, and treat GC based on the guidance of predictive biomarkers. To this end, a panel of GC cell lines was sequenced by a group of investigators and two major intrinsic subgroups of GC were identified. ${ }^{4}$ These subtypes were similar to the Lauren's intestinal and diffuse subtypes, so they were named genomic intestinal (G-INT) and genomic diffuse (G-DIF). The characteristics of these intrinsic subgroups reflect the diverse biologic behavior of GC, and their association with patient survival and treatment benefits are shown in Table 1. ${ }^{4,5}$

Following the advent of more comprehensive genomic assays, a new molecular classification system for the molecular and genomic basis of GC was proposed in 2014 by The Cancer Genome Atlas (TCGA) Consortium. This system classified GC into four major genomic subtypes: tumors positive for Epstein-Barr virus (EBV), microsatellite unstable tumors (MSI), genomically stable (GS), and tumors with chromosomal instability (CIN). ${ }^{6}$ For practical reason, more recently the Asian Cancer Research Group (ACRG) specifically divided GC into four subtypes: MSI, microsatellite stable (MSS)/epithelial-mesenchymal transition (EMT), MSS/tumor protein 53 (TP53) active, and MSS/TP53 inactive. $^{7}$ There are some differences between the two classification systems. The TCGA study evaluated 295 primary

Table I Intrinsic Gastric Cancer (GC) Subgroups Based on Gene Expression Pattern

\begin{tabular}{|c|c|}
\hline \multicolumn{2}{|c|}{$\begin{array}{l}\text { gene expression analysis of } 37 \mathrm{GC} \text { cell lines } \\
\text { two major intrinsic subgroups were identified } \\
\text { validated in primary tumors from } 52 \text { I patients (from Singapore, } \\
\text { Australia and South Korea) } \\
\text { the intrinsic genomic subtypes have } 64 \% \text { similarity to Lauren's } \\
\text { classification }\end{array}$} \\
\hline G-INT & G-DIF \\
\hline $\begin{array}{l}\text { Genes related to carbohydrate } \\
\text { and protein metabolism (FUT2) } \\
\text { and cell adhesion (LGALS4, } \\
\text { CDHI7) up-regulated }\end{array}$ & $\begin{array}{l}\text { Cell proliferation (AURKB) and } \\
\text { fatty acid metabolism (ELOVL5) } \\
\text { functional annotations were } \\
\text { enriched }\end{array}$ \\
\hline $\begin{array}{l}\text { G-INT tumors had superior } \\
\text { overall survival compared with } \\
\text { patients with G-DIF tumors }\end{array}$ & Worse survival outcomes \\
\hline $\begin{array}{l}\text { G-INT cell lines were significantly } \\
\text { more sensitive to } 5 \text {-FU and } \\
\text { oxaliplatin in vitro } \\
\text { More resistant to cisplatin }\end{array}$ & $\begin{array}{l}\text { G-DIF cell lines were more } \\
\text { sensitive to cisplatin }\end{array}$ \\
\hline
\end{tabular}

Abbreviations: G-INT, genomic intestinal; G-DIF, genomic diffuse.
GCs from Europe and the United States, while the ACRG system examined 300 primary GCs from a single center in South Korea. ${ }^{8}$ Next-generation sequencing (NGS) technology was used in the TCGA molecular classification, while immunohistochemistry (IHC) was used in the ACRG molecular classification to reduce costs and make it more practical. ${ }^{8}$ ACGR subtypes had significant survival differences, unlike the TCGA subtypes. The clinical correlations of each of the four subtypes of GC as classified by the TCGA and the ACGR are shown in Table 2.,8,9

Infectious agents, including the bacterium Helicobacter pylori and the Epstein-Barr virus (EBV), play an important role in GC. Infection has gradually been recognized as a major driver of inflammation-induced tumorigenesis and is thus a preventable cause of GC. Gastroesophageal reflux disease (GERD) is also considered an important risk factor for upper GC. Major processes implicated by frequently mutated genes associated with inflammation and cancer in three organs (stomach, colon, and liver) were extracted from molecular profiling databases by Guo et al, who established a multiscale model of the long-term evolutionary dynamics that lead from inflammation to tumorigenesis. ${ }^{10}$ The authors suggested that their findings provided a method for quantifying cancer risk and for the discovery of pathways driving inflammation-induced tumorigenesis, which could be used in the early detection and prevention of GC and the development of new treatment regimes. Zhang et al performed a single-cell transcriptomic study on gastric antral biopsies from patients with a variety of premalignant gastric lesions (ie chronic atrophic gastritis and intestinal metaplasia) and early gastric cancer (EGC). ${ }^{11}$ EGC is a lesion that is confined to the mucosa and submucosa regardless of lymph node involvement, and has a survival rate of $>90 \%$ at 5 years. The authors proposed that since some genes were not expressed in premalignant lesions but were prominently expressed in early and advanced gastric cancer cells, they can be used as cancer cell-specific molecular markers and precisely correlate with the earliest stages of GC tumorigenesis.

In most cases, $\mathrm{GC}$ is diagnosed at the metastatic or unresectable stage. Despite the latest improvements in GC treatment options, advanced GC remains one of the hardest to cure cancers and has a poor prognosis, with a median overall survival (OS) of 10-12 months and a 5-year OS of approximately $5-20 \%{ }^{12}$ There is therefore an unmet need for further efforts to palliate disease-related symptoms, improve patient quality of life, increase response rate and prolong progression free and overall survival while balancing the toxicities of therapy. 
Table 2 Comparison of Each Four Subtypes of Gastric Cancer (GC) According to TCGA or ACGR

\begin{tabular}{|c|c|}
\hline TCGA Subtypes & ACRG Subtypes \\
\hline Tumor samples from 295 treatment-naive primary GC patients & $\begin{array}{l}\text { mRNA expression level } \\
\text { analyzed in } 300 \text { tumor samples }\end{array}$ \\
\hline $\begin{array}{l}\text { Whole-exome sequencing, copy number analysis, messenger } \\
\text { ribonucleic acid sequencing, microRNA sequencing, DNA } \\
\text { methylation profiling and reverse-phase protein array analyses } \\
\text { were done }\end{array}$ & $\begin{array}{l}\text { Molecular subtypes have distinct prognostic } \\
\text { significance }\end{array}$ \\
\hline $\begin{array}{l}\text { EBV (+) GC } \\
\text { This subgroup represents } 9 \% \text { of all tumors and located } \\
\text { more frequently in the corpus and fundus } \\
\text { Has significant CIMP phenotype } \\
\text { Highest rate }(80 \%) \text { of PIK3CA mutations, } \\
\text { Increased rate of ARIDIA and BCOR mutations, } \\
\text { Over-expressions of PD-LI/2, JAK2, and ERBB2 } \\
\text { CDKN2A silencing }\end{array}$ & $\begin{array}{l}\text { MSS/TP53+ GC } \\
26 \% \text { of the total samples } \\
\text { frequent mutations in APC, ARIDIA, } \\
K R A S, P I K 3 C A \text {, and SMAD } 4 \\
\text { EBV infection is more frequent } \\
\text { intermediate prognosis }\end{array}$ \\
\hline $\begin{array}{l}\text { Microsatellite instability (MSI) GC } \\
\text { Constitutes } 22 \% \text { of total samples } \\
\text { associated with Helicobacter pylori (HP) infection and } \\
\text { intestinal metaplasia } \\
\text { silencing of MLHI gene, resulting in genomic instability } \\
\text { TP53, KRAS, PIK3CA, EGFR, ERBB2 and ERBB3, } \\
\text { ARIDIA mutations can be seen } \\
\text { a high rate of PD-Ll expression } \\
\text { a predilection for antral location } \\
\text { diagnosed at older ages (median } 72 \text { years) } \\
\text { more frequently seen in females (56\%) }\end{array}$ & $\begin{array}{l}\text { MSI-high GC } \\
23 \% \text { of total samples } \\
\text { intestinal histology (> } 60 \% \text { Lauren intestinal type) } \\
\text { diagnosed at early stages (>50\%) } \\
\text { ARIDIA }(44.2 \%) \text {, the PI3K-PTEN-mTOR pathway }(42 \%), \text { KRAS }(23.3 \%) \text {, } \\
\text { and ALK }(16.3 \%) \text { mutations } \\
\text { the best overall prognosis (can be diagnosed at early stages) and } \\
\text { lowest frequency of recurrence }(22 \%)\end{array}$ \\
\hline $\begin{array}{l}\text { Chromosomal instability (CIN) GC } \\
\text { Represents } 50 \% \text { of the total samples } \\
\text { Located at gastroesophageal junction or cardia (65\%) } \\
\text { Most commonly has intestinal histology } \\
\text { Activation of receptor tyrosine kinases-Ras (RTK/RAS) pathway } \\
\text { Amplifications of the genes ERBB2, KRAS/NRAS, EGFR, ERBB3, FGFR2, } \\
\text { MET and genes encoding cell cycle mediators, such as } \\
\text { cyclins EI, DI (CCNEI, CCNDI) and CDK6 } \\
\text { High frequency of TP53 mutations (73\%) }\end{array}$ & $\begin{array}{l}\text { MSS/TP53- GC } \\
36 \% \text { of the total samples } \\
\text { the highest frequency of TP53 mutations (60\%), frequent RHOA } \\
\text { mutations } \\
\text { ERBB2, EGFR, CCNEI, CCNDI, MDM2, ROBO2, GATA6 and MYC } \\
\text { enriched }\end{array}$ \\
\hline $\begin{array}{l}\text { Genomically stable (GS) GC } \\
20 \% \text { of the tumours } \\
\text { young patients (median } 59 \text { years) } \\
\text { the diffuse histological variant is common } \\
\text { cell adhesion and angiogenesis related pathways up-regulated: } \\
\text { E-cadherin }(C D H I) \text { (with the highest percentage) } \\
\text { rare TP53 mutations } \\
\text { Ras homolog family member A (RHOA) recurrent mutations } \\
\text { CLDNI8-ARHGAP fusions }\end{array}$ & $\begin{array}{l}\text { MSS/EMT GC } \\
\% \text { I5 of the tumours } \\
\text { diagnosed at younger age } \\
\text { diffuse type histology ( }>80 \%) \text { and signet ring cell carcinomas } \\
\text { a lower number of mutation events } \\
\text { the worst prognosis and the highest recurrence frequency }(63 \%) \\
\text { diagnosed at advanced stages (III/IV) } \\
\text { loss of CDHI expression is common frequent peritoneal seeding } \\
(64.1 \%)\end{array}$ \\
\hline
\end{tabular}

Abbreviations: TCGA, the cancer genome atlas; ACGR, asian cancer research group; CIMP, CPG island methylator phenotype; PIK3CA, phosphatidylinositol 3-kinase; ARIDIA, AT-rich interactive domain-containing protein IA; BCOR, B-cell lymphoma 6 corepressor; ERBB2, Erb-B2 receptor tyrosine kinase 2; PD-LI/2, programmed death ligand-1/2; JAK2, Janus associated kinase 2; MSI, microsatellite instability; CIN, chromosomal instability; CDK6, cell division protein kinase 6. 
Recent genome-scale sequencing studies have identified a plethora of genomic alterations and the activation of numerous molecular pathways in GC, including Human Epidermal Growth Receptor 2 (HER2), epidermal growth factor receptor (EGFR), fibroblast growth factor receptor-2 (FGFR2), mesenchymal epidermal transforming factor receptor (MET), the phosphatidylinositol 3-kinase (PI3K)/ mammalian target of rapamycin (mTOR), microsatellite instability (MSI), which are responsible for the complex heterogeneity of this disease. However, it is challenging to distinguish driver mutations from passenger mutations. Furthermore, hypermutated tumors (ie MSI or POLE mutant) more commonly include passenger mutations due to their inherent genetic instability. ${ }^{13}$ At this point, it is important to determine if the detected mutations are directly druggable oncogenic targets. Efforts to validate the therapeutic effects of the inhibition of some altered pathways have failed to yield a clinically meaningful outcome except for the identification of the overexpression/amplification of HER2, from which HER2 inhibition has significantly impacted clinical practice. Ultimately, the biomarkers that are available to guide the effective treatment of patients with advanced GC are HER2 overexpression, MSI/PD-L1 status and FGFR alterations.

HER2 (also known as erythroblastosis oncogene B2, ERBB2) is a proto-oncogene that encodes the transmembrane receptor-like HER2 protein. Its abnormally induced tyrosine kinase activity initiates signaling pathways that lead to cell proliferation, differentiation, and vascular and lymphatic angiogenesis. The reported rates of HER2 amplification in patients with GC range from 7 to $34 \% .{ }^{14-16}$ HER2 positivity also varies by tumor location (GEJ $>$ gastric body), histologic subtype (intestinal $>$ diffuse) and tumor grade (moderately differentiated $>$ poorly differentiated). ${ }^{17}$ HER2 overexpression is associated with the CIN subgroup according to the TCGA classification and with the MSS/TP53 inactive subtype according to the ACRG categorization. Both molecular subgroups demonstrate widespread genomic instability, which could explain the significant number of copy variations in major oncogenic drivers such as HER2. Although HER2 overexpression was shown to be associated with a poor prognosis in the most current and comprehensive meta-analysis to investigate the correlation between clinicopathologic characteristics and the prognostic significance of HER 2 expression in GC patients, ${ }^{18}$ the overall body of the literature regarding the prognostic significance of HER2 status in GC remains unsettled. ${ }^{19-21}$ Factors responsible for these inconsistencies include the use of different IHC staining methods, the lack of a uniform criteria for defining HER2 positivity, the potential influence of confounders in clinical studies (ie patient selection bias leading to unique study group characteristics compared with the general population or an insufficient observation period for calculating survival outcome) and the heterogeneous inherent characteristics of HER2 positive GC that may represent a specific intrinsic subtype that harbors unique genetic alterations. The design criteria of these studies are heterogeneous, and it should be kept in mind that most HER2-positive GC patients did not receive anti-HER2-directed treatment before the ToGA trial. The overall prognostic role of HER2 may also depend on tumor stage. The literature should therefore be interpreted with consideration of potential bias and the variation criteria for determining HER2 status.

\section{The Fundamental Role of Trastuzumab in Advanced GC}

A panel of murine monoclonal antibodies ( $\mathrm{mAb}$ ) capable of specifically inhibiting HER2-positive cell lines has been developed, of which muMAb 4D5 was the most potent. A humanized form of muMAb 4D5, trastuzumab has three times stronger binding affinity for HER 2 than its parent and is a therapeutic $\mathrm{mAb}$ that targets the extracellular domain (ECD) of HER2. ${ }^{22}$ Breast cancer xenograft experiments were the first to show that trastuzumab has anti-tumor activity. Several subsequent pivotal clinical trials revealed the tremendous impact of trastuzumab on the clinical management of HER2 positive breast cancer, and trastuzumab is now the standard of care at all treatment steps for these patients. After the identification of HER2 amplification in GC, the clinical efficacy of trastuzumab was evaluated in the treatment of advanced gastric or gastroesophageal junction (GEJ) adenocarcinoma in the global, Phase 3 TOGA trial. Enrolled patients were randomized to receive either trastuzumab plus chemotherapy (fluoropyrimidine plus cisplatin) or chemotherapy alone as first-line therapy. The OS benefit of adding trastuzumab to frontline chemotherapy has been shown in patients with HER2-positive advanced GC. Post hoc exploratory analyses of the TOGA trial demonstrated a 4.2-month improvement in median OS with trastuzumab in patients who expressed high HER2 levels (2+) on IHC $2+$ and fluorescence in situ hybridization (FISH) amplified HER $2 /$ CEP17 $\geq 2$ or IHC $3+$ (hazard ratio $(H R)=0.65$ ) compared with those with a low expression of HER2 protein and an IHC of 0 or $1+$ despite FISH positivity. ${ }^{23}$ Given the 
results of TOGA, assessment of tumor HER2 overexpression using IHC and FISH or some other in situ hybridization (ISH) method is recommended for all patients with inoperable locally advanced, recurrent, or metastatic gastric adenocarcinomas, and trastuzumab-containing regimens are now a standard option for the first-line treatment of patients with tumors that express HER2.

GC is a disease of aging, and there are several important and potentially life-threatening drawbacks to combination chemotherapy in the elderly population. In a meta-analysis including available data from 41 studies, HER2 positivity was not correlated with age. ${ }^{18}$ Prior literature on this relationship is controversial. ${ }^{24-26}$ Several early phase and retrospective studies evaluated the efficacy and tolerability of adding single-agent chemotherapy (ie lower dose capecitabine, cisplatin, or S-1) to trastuzumab in elderly patients with HER2positive advanced gastric cancer (AGC). The results of these studies were comparable to the ToGA trial endpoints in terms of overall survival and response rates. ${ }^{27-29}$ While a consensus has yet to be achieved, the general body of the literature suggests that trastuzumab enhances the antitumor activity of chemotherapy, and that the combination of singleagent chemotherapy and trastuzumab is a logical and safe option for elderly patients with HER2-positive AGC.

\section{Failures of HER2-Targeted Therapy in Advanced GC}

The Phase III HELOISE randomized study compared high dose (HD) trastuzumab plus chemotherapy with standard-ofcare trastuzumab plus chemotherapy as first-line management of HER2-positive metastatic gastric or gastroesophageal junction (GEJ) adenocarcinoma to investigate if HD trastuzumab increases the serum trough concentration of trastuzumab and improves OS. ${ }^{30}$ Although the serum trough concentration of trastuzumab was significantly increased in the HD trastuzumab group, no improvement in OS or progression-free survival (PFS) was observed.

Lapatinib, a dual EGFR and HER2 tyrosine kinase inhibitor, is approved as a second-line therapy for HER2-positive breast cancer. However, in the phase 3 LOGiC trial, the addition of lapatinib to capecitabine plus oxaliplatin as first-line therapy for HER2-positive advanced or metastatic esophageal, gastric or GEJ adenocarcinoma failed to achieve its primary OS endpoint despite a significant improvement in PFS and overall response rate (ORR). ${ }^{31}$ Possible explanations for these negative results include the selection of patients with HER2 overexpression based on FISH alone that $23 \%$ of patients had a prior gastrectomy, which could reduce lapatinib absorption, and the lack of antibody-dependent, cell-mediated cytotoxicity in the absence of trastuzumab.

Pertuzumab, is a recombinant humanized $\mathrm{mAb}$ that inhibits HER2 heterodimerization with HER3. The HER2/HER3 signaling dimer has been shown to be the most potent. ${ }^{32}$ Combining trastuzumab with pertuzumab is an effective strategy for patients with HER2+ breast cancer. Preclinical studies noted improved anti-tumor activity with dual HER2-blockade (pertuzumab and trastuzumab) compared with pertuzumab monotherapy in a xenograft model of HER2-positive GC. ${ }^{33}$

The JACOB trial evaluated the effectiveness of pertuzumab in combination with trastuzumab plus chemotherapy in patients with metastatic gastric or GEJ cancer. Although both PFS and ORR favored the pertuzumab-containing arm, no statistically significant difference was found in OS. ${ }^{34}$ Given the effectiveness of trastuzumab in the treatment of breast cancer, the benefits of continuing anti-HER2 therapy after progression while on trastuzumab have been studied in patients with HER2-positive AGC, with heterogeneous results. $^{35-37}$ In the Phase 2 T-ACT trial, patients with advanced HER2+ gastroesophageal adenocarcinoma (GEA) were randomized to receive weekly paclitaxel alone or in combination with trastuzumab as second-line therapy. ${ }^{38}$ Trastuzumab in combination with paclitaxel beyond progression failed to improve PFS (primary end point), and no beneficial biomarkers were identified. Tumor biopsy samples were tested for HER2 status both prior to enrollment and after disease progression in first-line trastuzumab-containing therapy, and it was found that HER2 amplification was lost in 11 of 16 patients (69\%). Additionally, in a subgroup analysis of this study, patients with longer trastuzumab-free intervals (>30 days) had a trend towards improved ORR, PFS, and OS in the paclitaxel plus trastuzumab group compared with paclitaxel alone.

Due to the unmet need for second-line treatment options in patients progressing on or after trastuzumab-based treatment, the benefits of adding HER2-targeted therapy as second-line therapy for advanced HER2-positive GC have been the subject of multiple clinical trials. Trastuzumab emtansine (T-DM1) was the first antibodydrug conjugate (ADC) to be composed of trastuzumab linked to emtansine, a tubulin inhibitor. Emtansine released into HER2-positive tumor cells causes mitotic arrest and apoptosis. T-DM1 has a role in the management of patients with both advanced and early stage HER2-positive breast cancer. The adaptive GATSBY phase II/III study assessed the role of 
T-DM1 as second-line therapy for GC. ${ }^{39}$ HER $2+$ GC patients $(\mathrm{IHC} 3+$ or $\mathrm{IHC} 2+$ and $\mathrm{FISH}+$ ) received either docetaxel or T-DM1. Trastuzumab was administered in $79 \%$ and $76 \%$ of the patients who received taxane and T-DM1, respectively, as part of first-line therapy. This trial did not favor T-DM1 compared with taxane.

In the phase 3 TyTAN trial, lapatinib was used as second-line therapy for HER2-positive AGC in the Asian population. Although a minority $(6 \%)$ of patients had previously received trastuzumab-containing therapy and the ORR was significantly higher with lapatinib plus paclitaxel versus paclitaxel alone, no OS or PFS benefit was derived from lapatinib treatment. ${ }^{40}$

When the results of these two studies are interpreted together, it appears that continuing anti-HER2 treatments once disease progression is noted based on initial HER2 status is not advised. However, the negative results of these works may be due to the temporal heterogeneity in HER2 expression being ignored. Pivotal clinical trials on HER2-positive GC are shown in Table 4.

\section{Mechanisms of Resistance to HER2-Targeted Therapies}

Repetitive failures after TOGA, unlike the response seen in breast cancer, highlight the distinct HER2 biology in GC. Unlike breast cancer, HER2 overexpression in gastric/GEJ adenocarcinomas tends to be more heterogeneous, both with respect to morphology and the immunoreactivity of tumor cells to antibodies detecting HER2. ${ }^{41,42}$ GCs are gland-forming, mucin-producing carcinomas that demonstrate incomplete basolateral or lateral staining patterns. ${ }^{42}$ Intra-tumor heterogeneity of HER2 expression occurs in 5 to $50 \%$ of cases depending on different definitions, leading to difficulties in assessing HER2 status in GCs. ${ }^{43}$ Table 3 summarizes a comparison of HER2 scoring systems for breast and gastric cancer according to the American Society of Clinical Oncology/College of American Pathologists (ASCO/CAP) 2013 guidelines and the scoring systems provided by Hoffman et al and Rüschoff et al. ${ }^{44-47}$

Discordance between FISH/IHC and next-generation sequencing (NGS) on intra-tumoral heterogeneity was recently reported. Four of 50 patients whose HER2 status was positive with FISH or IHC were shown to be negative for HER2 amplification by NGS and progressed rapidly on trastuzumab therapy. ${ }^{48}$ In addition to intra-tumoral heterogeneity, which can even be seen in the same tumor gland, discordant HER2 status between primary and metastatic disease is common in GC.

Several oncogenic alterations, such as phosphatase and tensin homolog (PTEN) deficiency, PI3K mutations, hyperactivation of the hepatocyte growth factor (HGF)/mesenchymal epithelial transition factor (MET) pathway, co-existing EGFR overexpression, and MET/KRAS amplifications can potentially reduce the growth-inhibitory effect of HER2-targeting drugs. ${ }^{49}$ This might also explain the variability in the overall response rates (between 32\% and 68\%) of patients treated with trastuzumab as first-line therapy even when trastuzumabbased treatment is the standard of care. ${ }^{50-52}$ This implies that not all patients benefit from trastuzumab, even in the presence of HER 2 expression. To this end, Gomez et al showed that the level of HER2 gene amplification significantly predicted the sensitivity of the tumor to trastuzumab therapy and the OS of AGCs treated with trastuzumab-based chemotherapy. ${ }^{53}$ The authors emphasized the importance of utilizing quantitative variables such as HER2 amplification ratio that can be objectively measured, rather than subjective factors such as IHC scores, which are not uniformly measured. On the other hand, tumors determined to be IHC $3+$ may not benefit from further information on HER2 gene amplification to reach a clinical decision on the use of trastuzumab-based treatment. ${ }^{52}$

Increased mRNA expression of EGFR, ERBB3, and ERBB4 were shown in a xenograft mouse model of trastuzumab-resistant GC. ${ }^{54}$ Data indicated that HER3 plays a role in tumor resistance to HER2 inhibitors and contributes to the proliferation of HER2-amplified cells through the activation of the PI3K-AKT pathway. ${ }^{55}$

HER2 status may be changed after trastuzumab-based treatment, leading to a reduced response to anti-HER2 treatment. Several studies have addressed the acquired loss of HER2 overexpression during therapy with anti-HER2 containing agents. ${ }^{56-58}$ It was found that the loss of HER2 positivity is more frequent in tumors with an initial IHC score of $2+{ }^{58}$ Further, Janjigian et al reported in their NGS analysis of tumor tissues from 44 patients after trastuzumab treatment that HER2 amplification was lost in $14 \%$ of samples in addition to other secondary variable alterations. ${ }^{48}$

Micro RNAs (miRNAs) are a class of non-coding RNAs that play an important role in the regulation of target genes at the post-transcriptional level and may therefore play a crucial role in regulating cancer biology. Transfection of a miRNA-125a precursor significantly enhanced trastuzumab inhibition of gastric cancer cell growth. There is growing evidence that several miRNAs regulate trastuzumab resistance through HER2 signaling 
Table 3 Summary of Characteristics of HER2 Scoring in Gastric and Breast Cancer

\begin{tabular}{|c|c|c|c|}
\hline & & Breast Cancer & Gastric Cancer \\
\hline $\begin{array}{l}\text { Immunohistochemistry } \\
\text { Scoring }\end{array}$ & & \multicolumn{2}{|l|}{$\begin{array}{l}\text { The first screening method for HER } 2 \text { evaluation } \\
\text { high concordance with FISH. Widespread method, } \\
\text { Easy, cheap, and quick. }\end{array}$} \\
\hline $\begin{array}{l}\text { HER2 expression in the } \\
\text { neoplastic cells }\end{array}$ & & $\begin{array}{l}\text { Membranous and predominantly } \\
\text { circumferential }\end{array}$ & $\begin{array}{l}\text { Basolateral or lateral, incomplete, } \\
\text { (usually not circular IHC staining) }\end{array}$ \\
\hline $\begin{array}{l}\text { Intratumoral } \\
\text { heterogeneity }\end{array}$ & & Not frequent & $\begin{array}{l}\text { Commonly seen, } \\
\text { focal or patchy positivity } \\
\text { Possible association between between } \\
\text { Helicobacter pylori bacterium and } \\
\text { HER2 intratumoral heterogeneity }\end{array}$ \\
\hline \multicolumn{4}{|l|}{ HER-2 IHC Scoring } \\
\hline IHC Score 0: & $\begin{array}{l}\text { Her } 2 \text { overexpression } \\
\text { negative by IHC }\end{array}$ & $\begin{array}{l}\text { No reactivity or membranous reactivity in less } \\
\text { than } 10 \% \text { of cells }\end{array}$ & No immunostaining \\
\hline IHC Score I+: & $\begin{array}{l}\text { Her } 2 \text { overexpression } \\
\text { negative by IHC }\end{array}$ & $\begin{array}{l}\text { Faint / visible membranous reactivity in more } \\
10 \% \text { of cells at } 40 \mathrm{X} \text { magnification/ detected in } \\
\text { only one part of the membrane }\end{array}$ & $\begin{array}{l}\text { Weak immunostaining in less than } 30 \% \\
\text { of tumor cells }\end{array}$ \\
\hline IHC Score 2+ & $\begin{array}{l}\text { Her } 2 \text { overexpression } \\
\text { equivocal by IHC } \\
\text { (Equivocal HER2 } \\
\text { expression by IHC to } \\
\text { be confirmed by FISH) }\end{array}$ & $\begin{array}{l}\text { Weak to moderate complete or basolateral } \\
\text { membranous reactivity in } \geq 10 \% \text { of tumour cells } \\
\text { (visible at } 10-20 X \text { magnification) }\end{array}$ & $\begin{array}{l}\text { Complete membranous staining, either } \\
\text { uniform or weak in } \geq 10 \% \text { of cells }\end{array}$ \\
\hline IHC Score 3+ & $\begin{array}{l}\text { Her } 2 \text { overexpression } \\
\text { positive }\end{array}$ & $\begin{array}{l}\text { Strong, complete basolateral or lateral } \\
\text { membranous reactivity in } \geq 10 \% \text { of tumour cells } \\
\text { (visible at } 2.5-5 X \text { magnification) }\end{array}$ & $\begin{array}{l}\text { Uniform intense membranous staining } \\
\text { in } \geq 30 \% \text { of cells }\end{array}$ \\
\hline FISH analysis & \multicolumn{3}{|c|}{$\begin{array}{l}\text { Objective and accurate } \\
\text { gold standard method } \\
\text { higher cost, need more time, equipment and specialist } \\
\text { fluorescent/silver/chromogenic/dual-color dual-hapten in situ hybridization can be used }\end{array}$} \\
\hline $\begin{array}{l}\text { HER2 gene copies: CEPI } 7 \\
\text { gene copies ratio for HER2 } \\
\text { overexpression }\end{array}$ & & $\begin{array}{l}\text { HER2/CEP I } 7 \text { ratio } \geq 2(2.2) \text {; positive } \\
\text { or the mean HER2 copy number was } \geq 6\end{array}$ & HER2/CEP 17 ratio $\geq 2$; positive \\
\hline Frequency & & $15-25 \%$ & $4.4-53.4 \%$ \\
\hline $\begin{array}{l}\text { Anatomic location of } \\
\text { the tumour }\end{array}$ & & No correlation & $\begin{array}{l}\text { More frequent at gastric cardia and } \\
\text { gastro-esophageal junction } \\
\text { adenocarcinoma and intestinal subtype }\end{array}$ \\
\hline Prognostic significance & & Unfavourable & Favorable/not fully established \\
\hline
\end{tabular}

Abbreviations: IHC, immunohistochemistry; CEP 17, chromosome 17 centromere.

pathway components and HER2 compensatory receptors, and that serum-based miRNA signature can effectively distinguish patients with HER2-positive advanced cancers who are sensitive to trastuzumab from those who are resistant. $^{59-61}$

\section{Novel Anti-HER2 Strategies to Overcome Trastuzumab Resistance and Future Perspectives}

New therapeutic agents and combination therapies beyond trastuzumab for the management of HER2-positive GC have 
Table 4 Pivotal Clinical Trials of HER2-Positive GC

\begin{tabular}{|c|c|c|c|c|c|c|c|}
\hline Trial & Phase & Agent & $\begin{array}{l}\text { Line of } \\
\text { Therapy }\end{array}$ & Region & ORR (\%) & Median PFS & Median OS \\
\hline ToGA & $\begin{array}{l}3 \\
(N=594)\end{array}$ & Trastuzumab & I st & Global & $\begin{array}{l}47 \text { vs } 35 \\
P=0.0017\end{array}$ & $\begin{array}{l}6.7 \text { vs } 5.5 \\
H R=0.7 I \\
P=0.0004\end{array}$ & $\begin{array}{l}I 3.8 \text { vs II. I } \\
H R=0.74 \\
P=0.0046\end{array}$ \\
\hline HELOISE & $\begin{array}{l}3 \\
(N=248)\end{array}$ & HD Trastuzumab & Ist & Global & $\begin{array}{l}56,9 \text { vs } 58.9 \\
P=0.76\end{array}$ & $\begin{array}{l}5.6 \text { vs } 5.7 \\
H R=1.04 \\
P=0.8222\end{array}$ & $\begin{array}{l}10.6 \text { vs } 12.5 \\
H R=1.24 \\
P=0.240 I\end{array}$ \\
\hline LOGIC & $\begin{array}{l}3 \\
(N=487)\end{array}$ & Lapatinib & Ist & Global & $\begin{array}{l}53 \text { vs } 39 \\
P=0.0031\end{array}$ & $\begin{array}{l}6.0 \text { vs } 5.4 \\
H R=0.82 \\
P=0.08 I\end{array}$ & $\begin{array}{l}I 2.2 \text { vs } 10.5 \\
H R=0.9 \mid \\
P=0.35\end{array}$ \\
\hline JACOB & $\begin{array}{l}3 \\
(N=780)\end{array}$ & Pertuzumab & Ist & Global & $\begin{array}{l}56.7 \text { vs } 48.9 \\
P=0.026\end{array}$ & $\begin{array}{l}8.5 \text { vs } 7.0 \\
P=0.0001\end{array}$ & $\begin{array}{l}\mid 7.5 \text { vs } \mid 4.2 \\
H R=0.84 \\
P=0.057\end{array}$ \\
\hline T-ACT & $\begin{array}{l}2 \\
(N=89)\end{array}$ & Paclitaxel plus trastuzumab & 2nd & Japan & $\begin{array}{l}33.3 \text { vs } 32 \\
P=1.00\end{array}$ & $\begin{array}{l}3.7 \text { vs } 3.2 \\
H R=0.91 \\
P=0.33\end{array}$ & $\begin{array}{l}10.2 \text { vs } 10 \\
H R=1.23 \\
P=0.20\end{array}$ \\
\hline GATSBY & $\begin{array}{l}2 / 3 \\
(N=345)\end{array}$ & TDM-I & 2nd & Global & $\begin{array}{l}20.6 \text { vs } 19.6 \\
P=0.84\end{array}$ & $\begin{array}{l}2.7 \text { vs } 2.9 \\
H R=1.13 \\
P=0.31\end{array}$ & $\begin{array}{l}7.9 \text { vs } 8.6 \\
H R=1.15 \\
P=0.86\end{array}$ \\
\hline TyTAN & $\begin{array}{l}3 \\
(N=26 I)\end{array}$ & Lapatinib & 2nd & Asia & $\begin{array}{l}27 \text { vs } 9 \\
P<0.00 I\end{array}$ & $\begin{array}{l}5.4 \text { vs } 4.4 \\
H R=0.85 \\
P=0.244\end{array}$ & $\begin{array}{l}I I \text { vs } 8.9 \\
H R=0.84 \\
P=0.10\end{array}$ \\
\hline
\end{tabular}

recently been studied. Pan-HER inhibitors may exert a better antitumor effect than HER2 blockade alone by blocking the activation of all HER family receptors, including heterodimers. Combining precision therapies may also overcome anti-HER2 treatment resistance in GC. Nam et al demonstrated the effectiveness of dacomitinib (an irreversible panHER tyrosine kinase inhibitor) in the inhibition of EGFR/ HER2, HER2/HER3, and HER3/HER4 heterodimer formation, as well as HER3 with p85 in a large panel of gastric cancer cell lines and noted synergy between dacomitinib and trastuzumab, IGF1R inhibitors, ERK1/2 inhibitors, and PI3K/mTOR inhibitors. ${ }^{55}$ A series of pan-HER TKIs, such as afatinib, dacomitinib, neratinib, and pyrotinib, have been tested for treatment effects against HER2-positive GC ${ }^{62,63}$ (SUMMIT, NCT01953926; NCT02500199).

Tucatinib (ONT 380), an oral HER2-targeted TKI with increased selectivity for HER2 compared with earliergeneration HER2-targeted TKIs, such as lapatinib and neratinib, was approved by the Food and Drug Administration (FDA) based on data from the pivotal HER2CLIMB trial for the treatment of patients with advanced HER2-positive breast cancer, including those with brain metastases, in combination with trastuzumab and capecitabine after at least one round of anti-HER2 treatment. ${ }^{64,65}$ Data showed improved response rates and central nervous system PFS $(\mathrm{HR}=0.32 ; 95 \% \mathrm{CI}, 0.22-0.48 ; \mathrm{P}<0.0001)$ in patients with HER2-positive brain metastatic breast cancer in the first year of therapy. ${ }^{66}$ Tucatinib demonstrated substantial antitumor activity in HER2-amplified esophageal and gastric cancers in preclinical studies. Hence, a Phase $1 \mathrm{~b}$ dose escalation study of tucatinib in combination with trastuzumab and oxaliplatin-based chemotherapy for HER2+ gastrointestinal cancers, and a randomized, double-blind, placebo-controlled, phase $2 / 3$ study of tucatinib in combination with trastuzumab, ramucirumab, and paclitaxel in patients with previously treated advanced HER2+ gastric or GEJ adenocarcinoma are ongoing (NCT04430738, NCT04499924, respectively,).

Until the DESTINY-Gastric01 study of trastuzumab deruxtecan, there were no other globally established 
HER2-directed agents for the treatment of GC that progressed after trastuzumab. Trastuzumab deruxtecan (T-DXd) (DS-8201a) is a novel anti-HER2 antibody-drug conjugate (ADC) that combines trastuzumab with a topoisomerase I inhibitor. In the international Phase II DESTINY-Gastric01 trial, patients with HER2-positive advanced gastric or gastroesophageal junction adenocarcinoma were randomly assigned T-DXd or the physician's choice of chemotherapy in a 2:1 ratio as third-line or later therapy. ${ }^{67}$

A significantly higher number of objective responses, a longer median duration of response (DOR) and prolonged OS were observed with T-DXd compared with chemotherapy. The confirmed ORR was $43 \%$ in the T-DXd group and $12 \%$ in the comparison group. Lower HER2 levels (IHC score of $2+$ with negative results on ISH or an IHC score of $1+$ ) seemed to correlate with a lower response rate (The confirmed ORR was $26.3 \%$ in the IHC score of $2+$ with negative results on ISH and $9.5 \%$ in the IHC score of 1+). ${ }^{68,69}$ This persistent but decreasing response might be due to the internalization of T-DXd by HER2-positive cells, the release of DXd into the cytoplasm of these cells and the transfer of the released DXd into adjacent HER2-negative cells (bystander effect). ${ }^{70,71}$ The high drug-to-antibody ratio of T-DXd and the membrane permeability of its payload may also make this ADC less dependent on a high level of HER2 expression. Following this study, the FDA approved T-DXd for use in advanced gastric/GEJ cancers after failure with a trastuzumab-containing regimen.

Interstitial lung disease and myelosuppression, which were mostly treated with dose reduction and interruption, were the only notable adverse events attributed to T-DXd. Interstitial lung disease developed in $10 \%$ of the patients in the T-DXd group, and 3 out of 12 cases were grade 3-4 adverse effects. Patients receiving T-DXd therefore require vigilant surveillance of pulmonary symptoms to avoid lethal lung toxicity.

Other HER2-targeted ADCs beyond T-DXd were evaluated in early clinical trials. In a phase II study, RC48ADC, a novel ADC comprised of a humanized anti-HER2 IgG1, a valine-citrulline linker and a microtubule inhibitor, MMAE, provided a clinically meaningful response and survival benefit in heavily pretreated patients with HER2overexpressing (IHC $2+$ or $3+$ ) gastric or GEJ cancers. ${ }^{72}$ The investigator-assessed confirmed ORR of the 127 included patients was $18.1 \%$ (95\% CI: $11.8 \%, 25.9 \%)$, and the median overall survival was 7.6 months $(95 \%$
CI: 6.6-9.2), with an acceptable safety profile. Phase 1 studies are ongoing to evaluate the safety, tolerability, and activity of Trastuzumab duocarmazine (known as SYD985), XMT-1522 (TAK-522), ARX788, ZW49, and other novel HER2-targeting ADCs in patients with advanced gastric tumors with variable HER2 expression levels.

ZW25 (Zanidatamab), a novel IgG1 bispecific antibody, targets two non-overlapping epitopes of HER2, in what is known as biparatopic binding, which results in dual HER2 signal blockade and enhanced receptor downregulation compared with trastuzumab. In the Phase I basket trial that was presented at the American Society for Clinical Oncology (ASCO) in 2018, single-agent ZW25 had a 56\% disease control (DCR) and 44\% ORR rate in patients with HER2positive gastroesophageal cancer that progressed on trastuzumab. ${ }^{73}$ Zanidatamab was well tolerated and toxicities were manageable. The third part of the trial evaluating the efficacy of ZW25 in combination with chemotherapy in the first-line treatment of HER2+ advanced GC and GEJ cancers is currently ongoing (NCT02892123). Zanidatamab was well tolerated and the toxicities were manageable. The third part of the trial evaluating the efficacy of ZW25 in combination with chemotherapy in the first-line treatment of HER2+ advanced GC and GEJ cancers is currently ongoing (NCT02892123).

Margetuximab is a novel investigational antibody derived from 4D5, the parent antibody of trastuzumab, that is designed to alter fragment crystallizable region (Fc) binding affinities. Fc engineering of margetuximab yielded increased affinity for the activating Fc $\gamma$ receptor (Fc $\gamma R$ ) CD16A (Fc $\gamma$ RIIIa) and decreased affinity for the inhibitory Fc $\gamma$ R CD32B (Fc $\gamma$ RIIb), which led to enhanced antibody-dependent cellular cytotoxicity (ADCC) against HER2-positive tumor cells, even at low HER2 expression levels. $^{74}$ The aim of margetuximab was also to potentiate innate and adaptive immunity and upregulate tumor PDL1 expression levels. Phase 1 study of margetuximab (MGAH22) found that single-agent margetuximab was well tolerated, with promising activity in heavily pretreated patients with HER2-expressing tumors. ${ }^{75}$

Data on the impact of immunotherapy on HER2+ GC continue to emerge. Trastuzumab has been found to stimulate HER2-specific $T$ cell response and increase tumor PD-L1 expression. ${ }^{76,77}$ Potential synergistic anti-tumor activity has been noted when anti-HER2 therapeutic approaches are combined with anti-PD-1 antibodies. In CP-MGAH22-05, a single-arm, multicenter, phase $\mathrm{Ib} / \mathrm{II}$ study, the combination 
of margetuximab and pembrolizumab, a selective humanized monoclonal immunoglobulin G4-Kappa antibody that binds to PD-1 and provokes an antitumor immune response, was administered to patients with advanced HER2+ gastric and gastroesophageal cancers who had received at least one previous treatment with trastuzumab plus chemotherapy. ${ }^{78}$ ORR was 18.48\% (95\% CI, 11.5-27.93), DCR was 53\% (95\% CI, $43-$ 64), median PFS by investigator assessment was 2.73 months (95\% CI, 1.61-4.34), and median OS was 12.48 months $(95 \%$ CI, 9.07-14.09). These results suggest that the combination of margetuximab and pembrolizumab might be an effective treatment option for GC or GEA while avoiding the toxicity of chemotherapeutic agents. Clinical outcomes were more pronounced in patients with HER2 IHC 3+, PD-L1-positive and HER2-amplified tumors assessed by circulating tumour DNA (ctDNA) analysis. This trial also suggests that HER2 amplification by ctDNA could be used to reassess HER2 status without the need to obtain a post-progression biopsy.

Due to the activity and safety profile observed in the CP-MGAH22-05 trial, the phase 2-3 MAHOGANY study is evaluating margetuximab in combination with checkpoint inhibitors (retifanlimab-Anti PD1 mAb, tebotelimabbispecific anti-PD1, and anti-lymphocyte activation gene 3 (LAG-3) mAb) with or without chemotherapy as first-line therapy for patients with HER2-positive advanced GEA (NCT04082364). ${ }^{79}$

In the phase $1 \mathrm{~b} / 2$ PANTHERA trial (NCT02901301), first-line triple therapy with pembrolizumab, trastuzumab and chemotherapy showed promising efficacy in the treatment of advanced HER2 amplified gastric cancer regardless of PD-L1 status. ${ }^{80}$ The ORR was $77 \%$, and patients with HER2 amplification per NGS ( $\geq 4$ copy number) had a statistically significantly longer median PFS than those without HER2 amplification (median PFS, 22.0 months vs 7.7 months; $\mathrm{P}=0.03$ ). The same trend was shown in patients with altered RTK/RAS pathways compared with wild-type RTK/RAS (median PFS, 13.8 months vs 4.9 months; $\mathrm{P}=0.001)$.

In another trial of the combination of pembrolizumab, trastuzumab and chemotherapy (oxaliplatin or cisplatin) in patients with HER-2-positive advanced EGA, the ORR was 91\% (32 of 35 patients; six CRs). ${ }^{81}$ PD-L1 expression and tumor mutation burden did not correlate with PFS, and no association was found between depth or duration of response and degree of DNA copy number alterations or the number of predicted strong clonal neoantigens. It was emphasized that none of the treated patients had tumors that were positive for high microsatellite instability or Epstein-
Barr virus, which are metastatic EGC subsets with a greater likelihood of response to anti-PD-L1 therapy. In this study, the success of combination therapy was repeatedly demonstrated regardless of PD-L1 expression. ${ }^{81,82}$ The researchers speculated that the induction of antibody-dependent cell-mediated cytotoxicity by trastuzumab improves the anti-tumor immune response by enhancing the presentation of tumor antigens. Future studies are needed to investigate the mechanism behind the synergistic benefits of combining pembrolizumab with trastuzumab and chemotherapy. The available evidence has suggested that pembrolizumab combined with trastuzumab and chemotherapy is a promising treatment option in patients with HER2-positive disease, and that correlative biomarkers found in the early-phase studies need to be validated by ongoing trials. The randomized, double-blind, placebo-controlled, global Phase III KEYNOTE-811 study to evaluate the efficacy and safety of pembrolizumab or placebo in combination with trastuzumab and chemotherapy as first-line treatment for patients with advanced HER2-positive GC or GEJ adenocarcinoma (NCT03615326) is still ongoing. ${ }^{83}$ The first interim results of the KEYNOTE-811 trial were presented at the 2021 ASCO Annual Meeting. Patients were included regardless of PD-L1 status, although $88 \%$ and $85 \%$ of those in the pembrolizumab and placebo (trastuzumab plus chemotherapy alone) arms at the interim timepoint, respectively, had a PD-L1 combined positive score $\geq 1$. The ORR of the pembrolizumab arm was $74.4 \%$, compared with $51.9 \%$ for the placebo arm $(p=0.00006)$. The addition of pembrolizumab also led to deeper responses, with $11 \%$ of patients in the pembrolizumab arm achieving a complete response compared with $3 \%$ in the placebo arm. In the interim safety analysis, although immune-mediated adverse events, particularly pneumonitis and colitis, were more common in the pembrolizumab group, the absence of new adverse events associated with combination treatment was highlighted. Adding pembrolizumab to the standard of care demonstrated a survival advantage for the first time since TOGA 11 years earlier. The practice-changing findings of the KEYNOTE-811 trial led the US FDA to grant accelerated approval of pembrolizumab in combination with trastuzumab and fluoropyrimidine and platinum-based chemotherapy as first-line therapy for patients with HER2-positive advanced GEA. KEYNOTE-811 is still recruiting, and overall survival and progression-free survival, which are the final primary endpoints of the study, are pending.

The multicenter phase II INTEGA trial was also designed to assess the efficacy, safety and tolerability of 
ipilimumab or 5-FU/folinic acid and oxaliplatin (FOLFOX) in combination with nivolumab and trastuzumab as first-line therapy for patients with advanced HER2positive EGC (NCT03409848). This ongoing study aims to evaluate the therapy options for advanced HER2positive $\mathrm{GC}$ in the first-line setting, including a chemotherapy-free experimental arm. In addition, the ability of immune profiling via liquid biopsy to identify predictive biomarkers to tailor treatment prior to initiation and before the second dose of nivolumab is under investigation. ${ }^{84}$

Based on previous data from studies that suggested a high overall response rate and manageable toxicities with T-DXd, the Phase $1 \mathrm{~b} / 2$ multi-center, open-label DESTINY-Gastric03 study is underway to investigate the safety, tolerability, pharmacokinetics, immunogenicity, and preliminary anti-tumor activity of trastuzumab deruxtecan alone and in combination with chemotherapy and/or durvalumab in HER2-positive advanced/metastatic gastric/ GEJ adenocarcinoma patients (NCT04379596). ${ }^{85}$

Due to the highly immunogenic nature of HER2 tumors, combining anti-HER2 therapies with immune checkpoint blockade is a high potential approach. An open-label, two cohort phase $1 \mathrm{~B} / 2$ study was designed to evaluate ZW25 plus chemotherapy with/without tislelizumab, an investigational anti-PD-1 antibody, as first-line therapy in patients with HER2-positive metastatic breast cancer (cohort 1) or advanced gastric/gastroesophageal junction adenocarcinoma (cohort 2). ${ }^{86}$ Safety, tolerability profile, and objective response rate are the primary endpoints of the study, which is ongoing. Novel HER2directed strategies are summarized in Table 5.

\section{Conclusion}

The only available biomarkers able to guide the effective treatment of advanced GC are HER2 overexpression, MSI/ PD-L1 status, and FGFR alterations. Various anti-HER2 agents have been evaluated after the success of the ToGA trial, but these failed to improve clinical outcomes significantly enough to merit the establishment of new options for HER2-targeted therapy in AGC. The recent practicechanging first interim findings of the KEYNOTE-811 trial led to the accelerated approval of pembrolizumab in combination with trastuzumab plus chemotherapy for patients with HER2-positive advanced gastric or GEJ cancer by the FDA, which is now a first-line therapy for GC. The combination of anti-HER $2 \mathrm{mAb}$ with immunotherapy appears to be a reasonable strategy for overcoming the immune
Table 5 Novel HER2-Directed Strategies

\begin{tabular}{|c|c|}
\hline Strategy & Selected Agents/Trial \\
\hline Antibody-drug conjugates & $\begin{array}{l}\text { Trastuzumab deruxtecan (DS- } \\
820 \text { la) } \\
\text { RC48-ADC (NCT03556345) }\end{array}$ \\
\hline $\begin{array}{l}\text { Monoclonal antibodies (with } \\
\text { augmented ADCC) }\end{array}$ & - Margetuximab \\
\hline Bispecific antibodies & $\begin{array}{l}\text { Tucatinib } \\
\text { Neratinib (+ trastuzumab or } \\
\text { cetuximab): (NCT03457896) }\end{array}$ \\
\hline $\begin{array}{l}\text { Immunotherapy } \\
\text { combinations }\end{array}$ & $\begin{array}{l}\text { KEYNOTE-8I I (NCT036I5326) } \\
\text { (Pembrolizumab or placebo + } \\
\text { trastuzumab +chemotherapy) } \\
\text { MAHOGANY (NCT04082364) } \\
\text { (Margetuximab } \pm \text { PD-I inhibitor } \pm \\
\text { chemotherapy } \pm \text { dual checkpoint } \\
\text { inhibitor) } \\
\text { INTEGA (NCT03409848) } \\
\text { (Ipilimumab or FOLFOX + nivolumab + } \\
\text { trastuzumab) } \\
\text { DESTINY-Gastric03 (NCT04379596) } \\
\text { (Trastuzumab deruxtecan } \pm \\
\text { chemotherapy } \pm \text { durvalumab) } \\
\text { NCT04276493 (ZW25 + } \\
\text { chemotherapy } \pm \text { tislelizumab) }\end{array}$ \\
\hline
\end{tabular}

Abbreviations: ADCC, antibody-dependent cellular cytotoxicity; PD-I, programmed death-I; ZW25, zanidatamab.

insensitivity of patients with AGC. T-DXd was also found to be effective in patients who had already received $\geq 2$ previous lines of treatment. As we know that assessing HER2 status in patients with GC is challenging due to their multiple heterogeneities, HER2 amplification level may represent a predictive biomarker for selecting patients who can benefit the most from HER2 targeted therapies, and ctDNA might serve as a more precise and noninvasive method for improving clinical outcomes. It is important to confirm over time that HER2 is still positive due to the loss of HER2 expression that can occur after anti-HER2-based treatment. Tumor NGS, ctDNA, and other biomarkers may clarify the population that can derive the most clinical benefit from anti-HER2 agents. It is expected that the optimal management of HER-2 positive GC will continue to evolve following further investigation.

\section{Disclosure}

The authors reported no conflicts of interest for this work. 


\section{References}

1. Bray F, Ferlay J, Soerjomataram I, et al. Global cancer statistics 2018: GLOBOCAN estimates of incidence and mortality worldwide for 36 cancers in 185 countries. CA Cancer J Clin. 2018;68 (6):394-424. doi:10.3322/caac.21492

2. Nagtegaal ID, Odze RD, Klimstra D, et al. The 2019 WHO classification of tumours of the digestive system. Histopathology. 2020;76 (2):182-188. doi:10.1111/his.13975

3. Lauren P. The two histological main types of gastric carcinoma: diffuse and so-called intestinal-type carcinoma. An attempt at a histo-clinical classification. Acta Pathol Microbiol Scand. 1965;64 (1):31-49. doi:10.1111/apm.1965.64.1.31

4. Tan IB, et al. Intrinsic subtypes of gastric cancer, based on gene expression pattern, predict survival and respond differently to chemotherapy. Gastroenterology. 2011;141(2):476-85, 485 e1-11. doi:10.1053/j.gastro.2011.04.042

5. Wang Q, Liu G, Hu C. Molecular classification of gastric adenocarcinoma. Gastroenterology Res. 2019;12(6):275-282. doi:10.14740/gr1187

6. Cancer Genome Atlas Research, N. Comprehensive molecular characterization of gastric adenocarcinoma. Nature. 2014;513 (7517):202-209. doi:10.1038/nature 13480

7. Cristescu R, Lee J, Nebozhyn M, et al. Molecular analysis of gastric cancer identifies subtypes associated with distinct clinical outcomes. Nat Med. 2015;21(5):449-456. doi:10.1038/nm.3850

8. Katona BW, Rustgi AK. Gastric Cancer Genomics: advances and Future Directions. Cell Mol Gastroenterol Hepatol. 2017;3 (2):211-217. doi:10.1016/j.jcmgh.2017.01.003

9. Wang Q, Xie Q, Liu Y, et al. Clinical characteristics and prognostic significance of TCGA and ACRG classification in gastric cancer among the Chinese population. Mol Med Rep. 2020;22(2):828-840. doi:10.3892/mmr.2020.11183

10. Guo Y, Nie Q, MacLean AL, et al. Multiscale modeling of inflammation-induced tumorigenesis reveals competing oncogenic and oncoprotective roles for inflammation. Cancer Res. 2017;77 (22):6429-6441. doi:10.1158/0008-5472.CAN-17-1662

11. Zhang P, Yang M, Zhang Y, et al. Dissecting the single-cell transcriptome network underlying gastric premalignant lesions and early gastric cancer. Cell Rep. 2019;27(6):1934-1947e5. doi:10.1016/j.celrep.2019.04.052

12. Thrift AP, El-Serag HB. Burden of gastric cancer. Clin Gastroenterol Hepatol. 2020;18(3):534-542. doi:10.1016/j.cgh.2019.07.045

13. Yuen ST, Leung SY. Genomics study of gastric cancer and its molecular subtypes. Adv Exp Med Biol. 2016;908:419-439.

14. Tanner M, Hollmén M, Junttila TT, et al. Amplification of HER-2 in gastric carcinoma: association with Topoisomerase Ilalpha gene amplification, intestinal type, poor prognosis and sensitivity to trastuzumab. Ann Oncol. 2005;16(2):273-278. doi:10.1093/annonc/mdi064

15. Gravalos C, Jimeno A. HER2 in gastric cancer: a new prognostic factor and a novel therapeutic target. Ann Oncol. 2008;19 (9):1523-1529. doi:10.1093/annonc/mdn169

16. Hofmann M, Stoss O, Shi D, et al. Assessment of a HER2 scoring system for gastric cancer: results from a validation study. Histopathology. 2008;52(7):797-805. doi:10.1111/j.13652559.2008.03028.x

17. Rajagopal I, et al. HER 2 Expression in Gastric and Gastro-esophageal Junction (GEJ) Adenocarcinomas. J Clin Diagn Res. 2015;9(3):EC06-10.

18. Lei -Y-Y, Huang J-Y, Zhao Q-R, et al. The clinicopathological parameters and prognostic significance of HER2 expression in gastric cancer patients: a meta-analysis of literature. World J Surg Oncol. 2017;15(1):68. doi:10.1186/s12957-017-1132-5

19. Gomez-Martin C, Garralda E, Echarri MJ, et al. HER2/neu testing for anti-HER2-based therapies in patients with unresectable and/or metastatic gastric cancer. J Clin Pathol. 2012;65(8):751-757. doi:10.1136/ jclinpath-2012-200774
20. Gu J, Zheng L, Wang Y, et al. Prognostic significance of HER2 expression based on trastuzumab for gastric cancer (ToGA) criteria in gastric cancer: an updated meta-analysis. Tumour Biol. 2014;35 (6):5315-5321. doi:10.1007/s13277-014-1693-7

21. Yildiz Y, Sokmensuer C, Yalcin S. Evaluation of c-Met, HGF, and HER-2 expressions in gastric carcinoma and their association with other clinicopathological factors. Onco Targets Ther. 2016;9:5809-5817. doi:10.2147/OTT.S107946

22. Harries M, Smith I. The development and clinical use of trastuzumab (Herceptin). Endocr Relat Cancer. 2002;9(2):75-85. doi:10.1677/ erc. 0.0090075

23. Bang Y-J, Van Cutsem E, Feyereislova A, et al. Trastuzumab in combination with chemotherapy versus chemotherapy alone for treatment of HER2-positive advanced gastric or gastro-oesophageal junction cancer (ToGA): a phase 3, open-label, randomised controlled trial. Lancet. 2010;376(9742):687-697. doi:10.1016/S0140-6736(10) 61121-X

24. Smolinska M, Grzanka D, Antosik P, et al. HER2, NF-к B, and SATB1 expression patterns in gastric cancer and their correlation with clinical and pathological parameters. Dis Markers. 2019;2019:6315936. doi:10.1155/2019/6315936

25. Roy PS, Nyodu T, Hazarika M, et al. Prevalence of HER2 expression and its correlation with clinicopathological parameters in gastric or gastroesophageal junction adenocarcinoma in North-East Indian population. Asian Pac J Cancer Prev. 2019;20(4):1139-1145. doi:10.31557/APJCP.2019.20.4.1139

26. Shabbir A, Qureshi M, Khalid A, et al. Gastric adenocarcinoma expressing human epidermal growth factor receptor in South Asian population. Saudi J Gastroenterol. 2018;24(5):289-293. doi:10.4103/ sjg.SJG_23_18

27. Kim YS, Sym SJ, Baek MY, et al. Low-dose capecitabine plus trastuzumab as first-line treatment in patients 75 years of age or older with HER2-positive advanced gastric cancer: a pilot study. Cancer Chemother Pharmacol. 2015;76(6):1267-1272. doi:10.1007/ s00280-015-2881-x

28. Kimura Y, Fujii M, Masuishi T, et al. Multicenter phase II study of trastuzumab plus S-1 alone in elderly patients with HER2-positive advanced gastric cancer (JACCRO GC-06). Gastric Cancer. 2018;21 (3):421-427. doi:10.1007/s10120-017-0766-x

29. Zhu B, Wu JR, Zhou XP, Retrospective A. Comparison of trastuzumab plus cisplatin and trastuzumab plus capecitabine in elderly her2-positive advanced gastric cancer patients. Medicine (Baltimore). 2015;94(34):e1428. doi:10.1097/MD.000000 0000001428

30. Shah MA, et al. HELOISE: phase IIIb randomized multicenter study comparing standard-of-care and higher-dose trastuzumab regimens combined with chemotherapy as first-line therapy in patients with human epidermal growth factor receptor 2-Positive Metastatic Gastric or Gastroesophageal Junction Adenocarcinoma. J Clin Oncol. 2017;35(22):2558-2567.

31. Hecht JR, Bang Y-J, Qin SK, et al. Lapatinib in combination with capecitabine plus oxaliplatin in human epidermal growth factor receptor 2-positive advanced or metastatic gastric, esophageal, or gastroesophageal adenocarcinoma: TRIO-013/LOGiC-a randomized Phase III trial. J Clin Oncol. 2016;34(5):443-451. doi:10.1200/ JCO.2015.62.6598

32. Tzahar E, Waterman $\mathrm{H}$, Chen X, et al. A hierarchical network of interreceptor interactions determines signal transduction by Neu differentiation factor/neuregulin and epidermal growth factor. Mol Cell Biol. 1996;16(10):5276-5287. doi:10.1128/MCB.16.10.5276

33. Yamashita-Kashima Y, Iijima S, Yorozu K, et al. Pertuzumab in combination with trastuzumab shows significantly enhanced antitumor activity in HER2-positive human gastric cancer xenograft models. Clin Cancer Res. 2011;17(15):5060-5070. doi:10.1158/ 1078-0432.CCR-10-2927 
34. Tabernero J, Hoff PM, Shen L, et al. Pertuzumab plus trastuzumab and chemotherapy for HER2-positive metastatic gastric or gastro-oesophageal junction cancer (JACOB): final analysis of a double-blind, randomised, placebo-controlled phase 3 study. Lancet Oncol. 2018;19(10):1372-1384. doi:10.1016/S1470-2045(18)30481-9

35. Palle J, Tougeron D, Pozet A, et al. Trastuzumab beyond progression in patients with HER2-positive advanced gastric adenocarcinoma: a multicenter AGEO study. Oncotarget. 2017;8(60):101383-101393. doi:10.18632/oncotarget.20711

36. Kawamoto Y, Meguro T, Yuki S, et al. Phase II study of trastuzumab with irinotecan in HER2-positive metastatic or advanced gastric cancer patients previously treated with trastuzumab and failed: HGCSG 1201/OGSG1205. J Clin Oncol. 2017;35(4_suppl):151. doi:10.1200/JCO.2017.35.4_suppl.151

37. Li Q, Jiang H, Li H, et al. Efficacy of trastuzumab beyond progression in HER2 positive advanced gastric cancer: a multicenter prospective observational cohort study. Oncotarget. 2016;7 (31):50656-50665. doi:10.18632/oncotarget.10456

38. Makiyama A, et al. Randomized, Phase II study of trastuzumab beyond progression in patients with HER2-positive advanced gastric or gastroesophageal junction cancer: WJOG7112G (T-ACT Study). J Clin Oncol. 2020;38(17):1919-1927. doi:10.1200/JCO.19.03077

39. Thuss-Patience PC, Shah MA, Ohtsu A, et al. Trastuzumab emtansine versus taxane use for previously treated HER2-positive locally advanced or metastatic gastric or gastro-oesophageal junction adenocarcinoma (GATSBY): an international randomised, open-label, adaptive, phase 2/3 study. Lancet Oncol. 2017;18(5):640-653. doi:10.1016/S1470-2045(17)30111-0

40. Satoh T, Xu R-H, Chung HC, et al. Lapatinib plus paclitaxel versus paclitaxel alone in the second-line treatment of HER2 -amplified advanced gastric cancer in asian populations: tyTAN - a randomized, Phase III Study. J Clin Oncol. 2014;32(19):2039-2049. doi:10.1200/ JCO.2013.53.6136

41. Satala CB, et al. HER2 Heterogeneity in Gastric Cancer: a Comparative Study, Using Two Commercial Antibodies. J Oncol. 2020;2020:8860174. doi:10.1155/2020/8860174

42. Oh DY, Bang YJ. HER2-targeted therapies - a role beyond breast cancer. Nat Rev Clin Oncol. 2020;17(1):33-48. doi:10.1038/s41571019-0268-3

43. Vakiani E. HER2 testing in gastric and gastroesophageal adenocarcinomas. Adv Anat Pathol. 2015;22(3):194-201. doi:10.1097/PAP.0000000000000067

44. Ruschoff J, Hanna W, Bilous M, et al. HER2 testing in gastric cancer: a practical approach. Mod Pathol. 2012;25(5):637-650. doi:10.1038/ modpathol.2011.198

45. Ruschoff J, Dietel M, Baretton G, et al. HER2 diagnostics in gastric cancer-guideline validation and development of standardized immunohistochemical testing. Virchows Arch. 2010;457(3):299-307. doi:10.1007/s00428-010-0952-2

46. Wolff AC, Hammond MEH, Hicks DG, et al. Recommendations for human epidermal growth factor receptor 2 testing in breast cancer: American Society of Clinical Oncology/College of American Pathologists clinical practice guideline update. Arch Pathol Lab Med. 2014;138(2):241-256. doi:10.5858/arpa.2013-0953-SA

47. Koopman T, Louwen M, Hage M, et al. Pathologic diagnostics of HER2 positivity in gastroesophageal adenocarcinoma. Am J Clin Pathol. 2015;143(2):257-264. doi:10.1309/AJCPCX69HGDDGYCQ

48. Janjigian YY, Sanchez-Vega F, Jonsson P, et al. Genetic predictors of response to systemic therapy in esophagogastric cancer. Cancer Discov. 2018;8(1):49-58. doi:10.1158/2159-8290.CD-17-0787

49. Palle J, Rochand A, Pernot S, et al. Human Epidermal Growth Factor Receptor 2 (HER2) in advanced gastric cancer: current knowledge and future perspectives. Drugs. 2020;80(4):401-415. doi:10.1007/ s40265-020-01272-5
50. Gravalos C, Gómez-Martín C, Rivera F, et al. Phase II study of trastuzumab and cisplatin as first-line therapy in patients with HER2-positive advanced gastric or gastroesophageal junction cancer. Clin Transl Oncol. 2011;13(3):179-184. doi:10.1007/ s12094-011-0637-6

51. Kurokawa Y, Sugimoto N, Miwa H, et al. Phase II study of trastuzumab in combination with S-1 plus cisplatin in HER2-positive gastric cancer (HERBIS-1). Br J Cancer. 2014;110(5):1163-1168. doi:10.1038/bjc.2014.18

52. Bang YJ, Yang OD, Kim SG, Kim WH. Gastric Cancer. In: Suayib Yalcin PAP, editor. Textbook of Gastrointestinal Oncology. Springer; 2019:73-95.

53. Gomez-Martin C, et al. Level of HER2 gene amplification predicts response and overall survival in HER2-positive advanced gastric cancer treated with trastuzumab. $J$ Clin Oncol. 2013;31 (35):4445-4452.

54. Sampera A, Sánchez-Martín FJ, Arpí O, et al. HER-family ligands promote acquired resistance to trastuzumab in gastric cancer. $\mathrm{Mol}$ Cancer Ther. 2019;18(11):2135-2145. doi:10.1158/1535-7163.MCT19-0455

55. Nam HJ, et al. Evaluation of the antitumor effects and mechanisms of PF00299804, a pan-HER inhibitor, alone or in combination with chemotherapy or targeted agents in gastric cancer. Mol Cancer Ther. 2012;11(2):439-451. doi:10.1158/1535-7163.MCT-11-0494

56. Seo S, Ryu M-H, Park YS, et al. Loss of HER2 positivity after anti-HER2 chemotherapy in HER2-positive gastric cancer patients: results of the GASTric cancer HER2 reassessment study 3 (GASTHER3). Gastric Cancer. 2019;22(3):527-535. doi:10.1007/ s10120-018-0891-1

57. Saeki H, Oki E, Kashiwada T, et al. Re-evaluation of HER2 status in patients with HER2-positive advanced or recurrent gastric cancer refractory to trastuzumab (KSCC1604). Eur J Cancer. 2018;105:41-49. doi:10.1016/j.ejca.2018.09.024

58. Pietrantonio F, Caporale M, Morano F, et al. HER2 loss in HER2-positive gastric or gastroesophageal cancer after trastuzumab therapy: implication for further clinical research. Int $J$ Cancer. 2016;139(12):2859-2864. doi:10.1002/ijc.30408

59. Nishida N, Mimori K, Fabbri M, et al. MicroRNA-125a-5p is an independent prognostic factor in gastric cancer and inhibits the proliferation of human gastric cancer cells in combination with trastuzumab. Clin Cancer Res. 2011;17(9):2725-2733. doi:10.1158/ 1078-0432.CCR-10-2132

60. Li H, Liu J, Chen J, et al. A serum microRNA signature predicts trastuzumab benefit in HER2-positive metastatic breast cancer patients. Nat Commun. 2018;9(1):1614. doi:10.1038/s41467-01803537-w

61. Mao L, Sun A-J, Wu J-Z, et al. Involvement of microRNAs in HER2 signaling and trastuzumab treatment. Tumour Biol. 2016;37 (12):15437-15446. doi:10.1007/s13277-016-5405-3

62. Sanchez-Vega F, Hechtman JF, Castel P, et al. EGFR and MET amplifications determine response to HER2 inhibition in ERBB2 amplified esophagogastric cancer. Cancer Discov. 2019;9 (2):199-209. doi:10.1158/2159-8290.CD-18-0598

63. Oh D-Y, Lee K-W, Cho JY, et al. Phase II trial of dacomitinib in patients with HER2-positive gastric cancer. Gastric Cancer. 2016;19 (4):1095-1103. doi:10.1007/s10120-015-0567-z

64. Kulukian A, et al. Preclinical Activity of HER2-selective tyrosine kinase inhibitor tucatinib as a single agent or in combination with trastuzumab or docetaxel in solid tumor models. Mol Cancer Ther. 2020;19(4):976-987. doi:10.1158/1535-7163.MCT-19-0873

65. Murthy RK, Loi S, Okines A, et al. Tucatinib, trastuzumab, and capecitabine for HER2-positive metastatic breast cancer. $N$ Engl J Med. 2020;382(7):597-609. doi:10.1056/NEJMoa1914609 
66. Lin NU, Borges V, Anders C, et al. Intracranial efficacy and survival with tucatinib plus trastuzumab and capecitabine for previously treated HER2-positive breast cancer with brain metastases in the HER2CLIMB trial. J Clin Oncol. 2020;38(23):2610-2619. doi:10.1200/JCO.20.00775

67. Shitara K, Bang Y-J, Iwasa S, et al. Trastuzumab Deruxtecan in previously treated HER2-Positive gastric cancer. $N$ Engl J Med. 2020;382(25):2419-2430. doi:10.1056/NEJMoa2004413

68. Yamaguchi K, Iwasa YB,S, Sugimoto N, et al., 1422MO Trastuzumab deruxtecan (T-DXd; DS-8201) in patients with HER2-low, advanced gastric or gastroesophageal junction (GEJ) adenocarcinoma: results of the exploratory cohorts in the Phase II, multicenter, open-label DESTINY-Gastric01 study, ESMO Virtual Congress 2020. 2020.

69. Shitara K, Baba E, Fujitani K, et al. Discovery and development of trastuzumab deruxtecan and safety management for patients with HER2-positive gastric cancer. Gastric Cancer. 2021;24(4):780-789. doi:10.1007/s10120-021-01196-3

70. Ogitani Y, Hagihara K, Oitate M, et al. Bystander killing effect of DS-8201a, a novel anti-human epidermal growth factor receptor 2 antibody-drug conjugate, in tumors with human epidermal growth factor receptor 2 heterogeneity. Cancer Sci. 2016;107(7):1039-1046. doi:10.1111/cas. 12966

71. Mitani S, Kawakami H. Emerging targeted therapies for HER2 positive gastric cancer that can overcome trastuzumab resistance. Cancers (Basel). 2020;12(2):2. doi:10.3390/cancers12020400

72. Peng Z, Liu T, Wei J, et al. A phase II study of efficacy and safety of RC48-ADC in patients with locally advanced or metastatic HER2-overexpressing gastric or gastroesophageal junction cancers. $J \quad$ Clin Oncol. 2020;38(15_suppl):4560. doi:10.1200/ JCO.2020.38.15_suppl.4560

73. Meric-Bernstam F, Beeram M, Mayordomo JI, et al. Single agent activity of ZW25, a HER2-targeted bispecific antibody, in heavily pretreated HER2-expressing cancers. J Clin Oncol. 2018;36 (15_suppl):2500. doi:10.1200/JCO.2018.36.15_suppl.2500

74. Rugo HS, Im S-A, Cardoso F, et al. Efficacy of margetuximab vs trastuzumab in patients with pretreated ERBB2-positive advanced breast cancer: a phase 3 randomized clinical trial. JAMA Oncol. 2021;7(4):573-584. doi:10.1001/jamaoncol.2020.7932

75. Bang YJ, Giaccone G, Im SA, et al. First-in-human phase 1 study of margetuximab (MGAH22), an Fc-modified chimeric monoclonal antibody, in patients with HER2-positive advanced solid tumors. Ann Oncol. 2017;28(4):855-861. doi:10.1093/annonc/mdx002

76. Kono K, et al. Impaired antibody-dependent cellular cytotoxicity mediated by herceptin in patients with gastric cancer. Cancer Res. 2002;62(20):5813-5817.

77. Chaganty BKR, Qiu S, Gest A, et al. Trastuzumab upregulates PD-L1 as a potential mechanism of trastuzumab resistance through engagement of immune effector cells and stimulation of IFNgamma secretion. Cancer Lett. 2018;430:47-56. doi:10.1016/j.canlet.2018. 05.009
78. Catenacci DVT, Kang Y-K, Park H, et al. Margetuximab plus pembrolizumab in patients with previously treated, HER2-positive gastro-oesophageal adenocarcinoma (CP-MGAH22-05): a single-arm, phase 1b-2 trial. Lancet Oncol. 2020;21(8):1066-1076. doi:10.1016/S1470-2045(20)30326-0

79. Catenacci DV, Rosales M, Chung HC, et al. MAHOGANY: margetuximab combination in HER2+ unresectable/metastatic gastric/gastroesophageal junction adenocarcinoma. Future Oncol. 2021;17 (10):1155-1164. doi:10.2217/fon-2020-1007

80. Rha SY, Lee C-K, Kim HS, et al. A multi-institutional phase Ib/II trial of first-line triplet regimen (Pembrolizumab, Trastuzumab, Chemotherapy) for HER2-positive advanced gastric and gastroesophageal junction cancer (PANTHERA Trial): molecular profiling and clinical update. J Clin Oncol. 2021;39(3_suppl):218. doi:10.1200/ JCO.2021.39.3_suppl.218

81. Janjigian YY, Maron SB, Chatila WK, et al. First-line pembrolizumab and trastuzumab in HER2-positive oesophageal, gastric, or gastro-oesophageal junction cancer: an open-label, single-arm, phase 2 trial. Lancet Oncol. 2020;21(6):821-831. doi:10.1016/ S1470-2045(20)30169-8

82. Janjigian YY, Chou JF, Simmons M, et al. First-line pembrolizumab $(\mathrm{P})$, trastuzumab (T), capecitabine (C) and oxaliplatin (O) in HER2-positive metastatic esophagogastric adenocarcinoma (mEGA). J Clin Oncol. 2019;37(4_suppl):62. doi:10.1200/ JCO.2019.37.4_suppl.62

83. Chung HC, Bang Y-J, S Fuchs C, et al. First-line pembrolizumab/ placebo plus trastuzumab and chemotherapy in HER2-positive advanced gastric cancer: KEYNOTE-811. Future Oncol. 2021;17 (5):491-501. doi:10.2217/fon-2020-0737

84. Tintelnot J, Goekkurt E, Binder M, et al. Ipilimumab or FOLFOX with Nivolumab and Trastuzumab in previously untreated HER2-positive locally advanced or metastatic EsophagoGastric Adenocarcinoma - the randomized phase 2 INTEGA trial (AIO STO 0217). BMC Cancer. 2020;20(1):503. doi:10.1186/s12885-02006958-3

85. Janjigian YY, Viglianti N, Liu F, et al. A phase Ib/II, multicenter, open-label, dose-escalation, and dose-expansion study evaluating trastuzumab deruxtecan (T-DXd, DS-8201) monotherapy and combinations in patients with HER2-overexpressing gastric cancer (DESTINY-Gastric03). J Clin Oncol. 2021;39(3_suppl):TPS261TPS261. doi:10.1200/JCO.2021.39.3_suppl.TPS261

86. Oh D-Y, Chung HC, Im YH, et al. ZW25, an anti-HER2 bispecific antibody, plus chemotherapy with/without tislelizumab as first-line treatment for patients with advanced HER2-positive breast cancer or gastric/gastroesophageal junction adenocarcinoma: a phase $1 \mathrm{~B} / 2$ trial-in-progress. J Clin Oncol. 2020;38(15_suppl):TPS3145TPS3145. doi:10.1200/JCO.2020.38.15_suppl.TPS3145
OncoTargets and Therapy

\section{Publish your work in this journal}

OncoTargets and Therapy is an international, peer-reviewed, open access journal focusing on the pathological basis of all cancers, potential targets for therapy and treatment protocols employed to improve the management of cancer patients. The journal also focuses on the impact of management programs and new therapeutic agents and protocols on patient perspectives such as quality of life, adherence and satisfaction. The manuscript management system is completely online and includes a very quick and fair peer-review system, which is all easy to use. Visit http://www.dovepress.com/ testimonials.php to read real quotes from published authors. 